\title{
Nikon.
}

\section{Nikon's large-format multiphoton system for intravital imaging}

Ultrafast resonant scanners; high-sensitivity, low-noise GaAsP detectors; and industry-leading optics have made Nikon's A1R MP multiphoton confocal microscope a system of choice for deep tissue imaging. Recent collaborative efforts between Nikon and the Allen Institute for Brain Science resulted in the design of an open-architecture multiphoton system able to image large specimens. Stimulated by this collaboration, Nikon has developed a commercially available large-format multiphoton system that can be used for the most demanding intravital imaging applications.

\section{Deep, fast, dual-color infrared imaging}

There is increasing demand for the ability to image deeper into tissues in in vivo and intravital imaging. Long-wavelength light can penetrate deeper into a sample than short wavelengths owing to decreased light scattering. Studies have also shown that there is considerably less photodamage at longer wavelengths. Nikon's A1R MP scanner and $25 \times / 1.1-N A$ (numerical aperture) multiphoton objective lens are optimized for use with long-wavelength laser sources (up to 1,300 nm). These elements, combined with high-sensitivity GaAsP non-descanned detectors, provide researchers with a multiphoton imaging system that can image deep into a sample, accommodate red-shifted fluorescent fluorophores such as mCherry, and minimize photodamage (Fig. 1). In addition, Nikon's latest iteration of the A1R MP allows for input of dualinfrared (IR) laser beams to accommodate simultaneous two-color IR imaging. It is also compatible with external photostimulation devices such as a digital micromirror device that enables flexible wavelength choices and simultaneous stimulation and imaging for advanced optogenetics experiments.

\section{Open-architecture A1R MP}

One of the major limitations of a traditional multiphoton system configured on a microscope stand is the maximum specimen size, which typically limits the choice of samples to ex vivo tissues or immobilized small animals such as rodents. To meet demands for more flexible sample choices for intravital imaging, researchers at the Allen Institute for Brain Science recently collaborated with Nikon to develop a flexible multiphoton system that can accommodate larger samples. The reconfigured Nikon A1R MP system has an open gantry-frame configuration that provides ample clearance for large

\section{Lynne Chang}

Nikon Instruments, Inc., Melville, New York, USA. Correspondence should be addressed to L.C. (Ichang@nikon.net) or Steve Ross (sross@nikon.net).

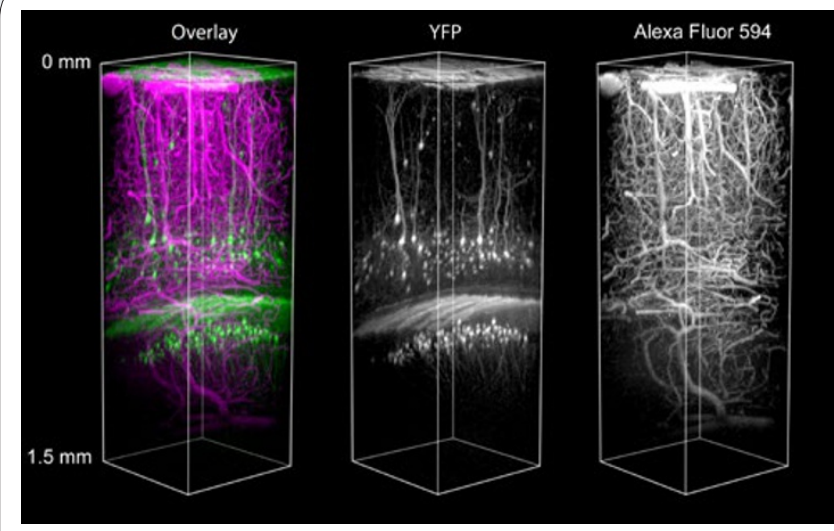

Figure 1 | In vivo dual-color imaging of mouse brain with Nikon's A1R MP. Shown is the cerebral cortex of an anesthetized YFP-H mouse (4 weeks old) imaged by the open-skull method. Alexa Fluor 594 was injected into the tail vein to visualize the blood vessels. A CFI75 Apochromat LWD 25×/1.10-NA MP1300 water-immersion objective lens and 1,100-nm excitation wavelength were used. Image courtesy of R. Kawakami, T. Hibi and T. Nemoto, Research nstitute for Electronic Science, Hokkaido University.

specimens (Fig. 2). The Allen Institute researchers plan to use this system for applications such as imaging the cortex of a mouse that is running on a turntable-style running wheel while being provided with visual stimuli through an adjacent video monitor. Stimuli will include a variety of drifting gratings, natural scenes, noises, etc., and readouts will typically involve two-photon time-lapse imaging combined with behavioral data such as running speed, eye tracking and body-posture evaluation. The long-term goal for the Allen Institute researchers is to generate a physiological atlas of neural activity in the mouse visual system underlying sensory representation, behavior and cognition, and to make the data freely and publicly available. 


\section{APPLICATION NOTES}

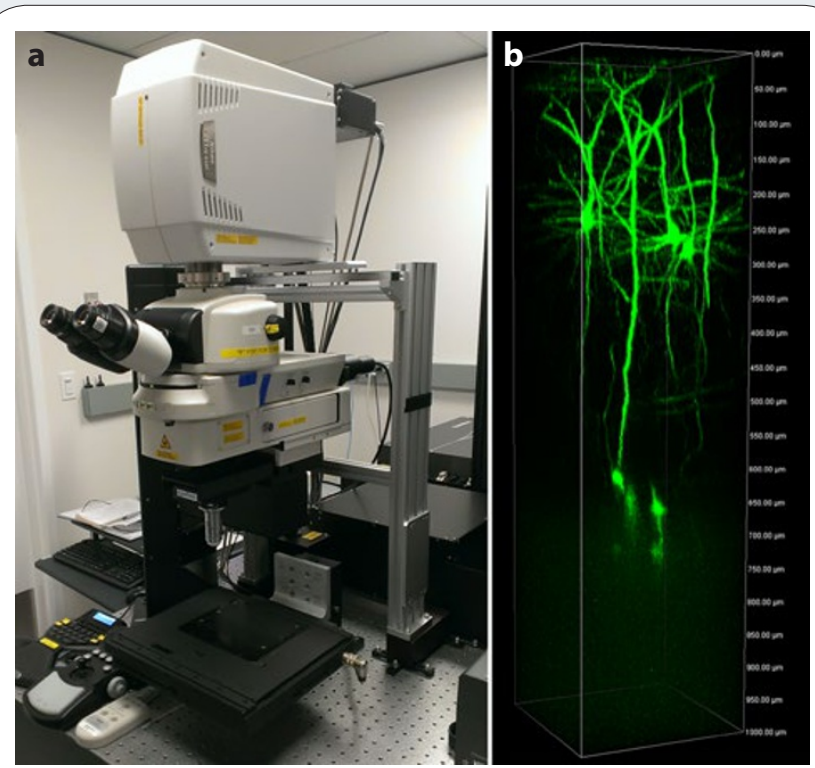

Figure 2 | A Nikon A1R MP configured on a gantry frame to accommodate intravital imaging of large specimens at the Allen Institute for Brain Science. (a) The instrument can be configured with (shown) or without a stage to accommodate a variety of samples. (b) A z-stack of cortex images from a Thy 1eGFP transgenic mouse captured with the new A1R MP design. A CFI75 LWD $16 \times / 0.8-N A$ water-immersion objective lens was used. Images courtesy of the Allen Institute for Brain Science.

Nikon's new large-format A1R MP for intravital imaging Stimulated by its collaborative efforts with the Allen Institute for Brain Science, Nikon has developed a new, commercially available largeformat multiphoton system. This large-format A1R MP is similar to the system described above, in that the multiphoton microscope is configured on a gantry frame, providing clear access all around the objective. A variety of stages can be accommodated if needed, or the system can be configured without a stage to provide extra room. This new A1R MP can easily image samples such as mice running on treadmills, and it can accommodate large stereotactic instruments and even larger specimens such as fetal pigs and monkeys, thereby expanding the application possibilities for multiphoton intravital imaging. These attributes, combined with the system's fast, sensitive, dual-IR imaging capabilities and ability to accommodate external photostimulation devices, make the new large-format A1R MP a flexible tool for custom intravital imaging applications. 